\section{Necesidades educativas especiales, elementos para una propuesta de inclusión educativa, a través de la investigación acción participativa. El caso de la escuela México}

Yasna Molina Olavarría*

\begin{abstract}
RESUMEN
El presente estudio tiene como propósito la elaboración, ejecución y desarrollo de un modelo de inclusión educativa, por la Investigación Acción Participativa, para la comunidad escolar de la Escuela México de la ciudad de Valdivia. La propuesta pretende crear, por medio de distintos elementos facilitadores de clases inclusivas, como estilos de aprendizaje, trabajo colaborativo entre parejas, colaboración entre profesionales, talleres para padres, entre otros componentes que surgen en el transcurso de la investigación. Permite, de esta forma, la inclusión de las alumnas que presentan Necesidades Educativas Especiales en el establecimiento en estudio. Además, por medio de la presente propuesta educativa, se pretende promover el desarrollo de una cultura inclusiva de enseñanza en toda la comunidad educacional, a través de la reflexión, evaluación y retroalimentación constante de los participantes, propia de la metodología de la Investigación Acción.
\end{abstract}

Palabras-claves: Inclusión. Integración. Diversidad. Necesidades Educativas Especiales.

\section{INTRODUCCIÓN}

L a presente investigación en términos generales, pretende construir un modelo de atención educativa que mejore las prácticas pedagógicas de la Comunidad Escolar de la Escuela México de la ciudad de Valdivia, frente a las Necesidades Educativas Especiales (en adelante NEE) que presentan las alumnas de acuerdo al enfoque teórico de Educación Inclusiva. El establecimiento educacional donde se llevó a cabo el estudio es una escuela pública, con dependencia municipal, que atiende en sus clases exclusivamente a estudiantes de género femenino. Presenta un índice de vulnerabilidad del $74 \%$ aproximadamente y una matrícula de 987 alumnas durante el año que se realizó la indagación.

Revista @rquivo Brasileiro de Educação, Belo Horizonte, vol.2, num.3, jan-jun, 2014.
* Universidad Austral de Chile, Escuela de Filosofía y Humanidades, Valdivia, Chile. Magister (c) en Educación Mención Gestión y Política Educativa. e-mail: yasnamusica@ gmail.com 
La educación inclusiva se basa en los mismos fundamentos de la educación regular, asentada en que todos los niños y niñas de una determinada comunidad aprendan juntos independientemente de sus condiciones personales, sociales o culturales. Siguiendo a Booth (2000), pensar la educación en forma inclusiva es preponderante en la sociedad actual. Por lo tanto, uno de los mayores desafíos de la educación es hacer efectivo el derecho que tienen todos los niños, niñas y jóvenes de acceder a una enseñanza de calidad y equidad para todos, que responda a sus necesidades individuales de aprendizaje, y a la vez, considerar una estructura curricular común (UNITED NATIONS EDUCATIONAL SCIENTIFIC AND CULTURAL ORGANIZATION, 2000).

Por lo anteriormente expuesto, por medio de la presente propuesta educativa, se pretende promover el desarrollo de una cultura inclusiva de enseñanza en toda la comunidad educacional, a través de la reflexión, evaluación y retroalimentación constante de los participantes, propia de la metodología de la Investigación Acción (KEMMIS; MCTAGGART, 1988; LATORRE, 2003).

Considerando la importancia de una educación más inclusiva, la presente investigación, pretende transformar una realidad educativa en torno a las NEE, cambiando el enfoque pedagógico de integración que existe hoy en sus prácticas, a uno más inclusivo, donde no existan realidades separadas para los niños y niñas.

\section{NECESIDADES EDUCATIVAS ESPECIALES}

Los alumnos con necesidades educativas especiales son aquellos cuyas necesidades educativas individuales no pueden ser resueltas con los medios y los recursos que habitualmente utiliza el docente para responder a las diferencias individuales de sus alumnos y que requieren para ser atendidas de ajustes, recursos o medidas pedagógicas especiales. De eso se infiere que el sistema educativo debe proveer los recursos humanos, técnicos y materiales necesarios para la equiparación de las oportunidades de los alumnos con necesidades educativas especiales, así como las orientaciones técnicas con el objeto de lograr aprendizajes de calidad (MINEDUC CHILE, 2005).

Revista @rquivo Brasileiro de Educação, Belo Horizonte, vol.2, num.3, jan-jun, 2014. 
Necesidades educativas especiales, elementos para una propuesta de inclusión educativa, a través de la investigación acción participativa. El caso de la escuela México

\section{ANTECEDENTES DE LA EDUCACIÓN ESPECIAL EN CHILE}

Las principales políticas de integración en Chile surgieron como una necesidad de buscar y asegurar la igualdad de oportunidades para todos los niños, niñas, jóvenes y adultos que presenten necesidades educativas especiales. En sus inicios, la educación especial estuvo enfocada a los alumnos con discapacidad y a mediados de los años 70 en adelante, también se orientó a niños y jóvenes que presentan dificultades de aprendizaje (CHILE, 2005).

Siguiendo a Godoy, Meza e Salazar (2004), desde que se comienza a debatir sobre la Educación Especial, ésta estuvo muy estrechamente ligada con las ciencias de la medicina y la psicología. A partir de este enfoque (medico-psicológico), se inició el estudio y descripción de los déficits, determinando las categorías clasificatorias en función de la etiología, con el principal objetivo de "curar o corregir", una condición deficitaria o patológica. Con el paso de los años y tomando como referencia el aporte psicológico, se buscó apoyar a los niños y jóvenes a partir de sus particularidades y del déficit diagnosticado y definido. Esas dos formas de ver las NEE tuvieron su mayor auge en los años 40 y 60, años en los que se construye una forma de atención de los niños con discapacidad, de carácter altamente segregativo, donde eran atendidos en centros e instituciones especiales, separadas de las escuelas regulares (UNITED NATIONS EDUCATIONAL SCIENTIFIC AND CULTURAL ORGANIZATION, 1994)

El concepto de Necesidades Educativas Especiales surge por primera vez en la década de los años 70 , en el denominado informe Warnock, para designar a las personas que presentan algún problema de aprendizaje, durante el periodo de su formación escolar, la que requiere de una atención más concreta y mayores recursos educativos y pedagógicos en comparación con el resto del grupo curso. De esa manera, por primera vez se considera a los sistemas educativos como responsables de responder a la diversidad, respetando las necesidades educativas de cada uno de los niños y niñas (CHILE, 2004a).

Posteriormente, especialmente en los últimos 30 años, se han desarrollado nuevos contextos de la Educación Especial. El vuelco más significativo fue el hecho de abandonar el enfoque médico, centrándose en apoyar específicamente lo educativo, lo que produjo mejoras en el proceso de enseñanza aprendizaje de la población que presenta

Revista @rquivo Brasileiro de Educação, Belo Horizonte, vol.2, num.3, jan-jun, 2014. 
necesidades educativas especiales, beneficiando de paso a toda la diversa población de estudiantes que componen el sistema educativo niñas (CHILE, 2004b)

Bajo esos fundamentos, los puntos de vista que consideran a la educación regular y especial como realidades separadas, iniciaron un proceso de cambio de enfoque, comprendiendo que los niños con necesidades educativas deben recibir una instrucción equitativa y apuntando a los fines que plantea la educación, poniendo a disposición de los niños, niñas y jóvenes un conjunto de recursos de apoyos especializados, para satisfacer las necesidades educativas especiales presente en la diversidad de alumnos que acogen las distintas unidades escolares.

\section{PRINCIPALES OBSTÁCULOS QUE PRESENTA EL SISTEMA EDUCATIVO CHILENO FRENTE A LAS NEE}

a) Uno de los factores de calidad de la educación actual está basado en resultados estandarizados, donde distintos diversos sistemas de evaluación, como el SIMCE (Sistema de Medición de Calidad de la Educación), miden las competencias adquiridas de los alumnos en un determinado nivel de su educación formal, eso conlleva a que toda la comunidad educativa de una institución escolar ponga su esfuerzo y energía en post de obtener un "buen resultado", que lo sitúe en un determinado lugar de un "ranking", lo que le aportará un nivel o estatus académico y económico, existiendo escuelas de excelencia, regulares y malas (FUNDO DAS NAÇÕES UNIDAS PARA A INFÂNCIA, 1999).

b) El sistema de medición de la calidad de la educación SIMCE no considera indicadores de calidad en relación a la enseñanza especial, trayendo como consecuencia la concentración de alumnos que presentan NEE en aquellos establecimientos más abiertos a la diversidad y que a su vez dan paso a la exclusión de estos alumnos de aquellos establecimientos que alcanzan mejores resultados (CHILE, 2004a)

b) El currículo de la educación en Chile presenta un enfoque homogéneo, donde no se realizan adaptaciones ni los ajustes curriculares de acuerdo a las necesidades y características específicas de los alumnos y alumnas.

Revista @rquivo Brasileiro de Educação, Belo Horizonte, vol.2, num.3, jan-jun, 2014. 
c) Otro obstáculo es el excesivo número de alumnos en las salas de clases, que afecta no sólo el acceso sino también la calidad de las respuestas educativas que requieren los estudiantes.

d) La Educación Especial no ha sido considerada en los procesos de Reforma Curricular. Como indica El Informe de la Comisión de Expertos en Educación Especial (CHILE, 200a), "A partir del año 1996, se fueron implementando progresivamente, los marcos curriculares de la Educación Básica, Media y Parvularia [...]. En las Bases Curriculares de Educación Parvularia se consideran las NEE de los alumnos, no así en el currículo de Educación Básica y Media". Los problemas que ha generado esta situación, es que los profesores en las distintas unidades educativas, no cuentan con las pautas o modelos para adaptar, ajustar o modificar el currículum escolar, para de esta forma responder a las características individuales que presentan cada uno de sus alumnos y alumnas, propio de la diversidad.

e) Otro obstáculo es la falta de criterios claros de promoción, certificación y egreso de los planes y programas de estudio de la educación especial, los cuales son incompatibles con los de educación regular, lo que produce que los niños y jóvenes con NEE presenten una desigualdad de oportunidades al momento de egresar o continuar estudios (CHILE, 2004a).

f) Los distintos docentes de educación común, durante su formación inicial, no fueron preparados para atender y responder a la diversidad desde la base de la formación regular. Además, por lo general los profesores se preparan o capacitan por motivación personal, sin considerar a la comunidad escolar en su conjunto (CHILE, 2004a).

g) Las familias de niños con NEE desconocen o no tienen redes que los apoyen y orienten. Además, las asociaciones de y para personas con discapacidad, no se encuentran verdaderamente organizadas para dar respuestas a sus problemas o dificultades. Por otra parte los padres y apoderados no son considerados en el proceso de enseñanza.

h) Bajo los puntos antes expuestos, es importante considerar realizar estudios e investigaciones, que propicien mejoras en las políticas y lineamientos entorno a las NEE, en función de realizar mejoras y cambios en los contextos escolares entorno a una educación hacia 
la diversidad, que entregue respuestas a cada uno de los niños y niñas, respetando sus características y capacidades personales.

\section{EDUCACIÓN PARA LA DIVERSIDAD. ¿INTEGRACIÓN O INCLUSIÓN?}

Teniendo en cuenta lo fundamental que es en la actualidad, el transformar e innovar desde y para la educación, partiendo desde una visión más integrista hacia una más inclusiva y que responda a las necesidades educativas y características de la diversidad de alumnas y alumnos que existentes en los contextos escolares, es necesario meditar y reflexionar en dichos modelos utilizados en las últimas décadas y que amparan a la gran mayoría de la población de estudiantes con dichas características.

Cuando se habla de integración e inclusión, lo habitual es utilizarlos como sinónimos, ya que semánticamente tienen significados muy parecidos. No obstante ambos términos representan realidades muy diferentes.

Las principales diferencias entre integración e inclusión en un sistema educativo son los siguientes: La integración es el mecanismo que busca realizar la inserción de las personas con NEE, creando mecanismos externos a la educación regular que reciben los demás niños del contexto escolar. En cambio en la inclusión, la escuela o unidades educativas se adaptan a las características individuales de los alumnos. Como señala Porras $(2005$, p. 50) "La integración se basa en la normalización de la vida del alumnado con necesidades específicas de apoyo educativo; sin embargo, la inclusión se presenta como un derecho de todos los individuos, presenten o no algún tipo de necesidad".

La integración se focaliza en apoyar educativamente a los alumnos con necesidades educativas específicas, para ello se desarrollan innovaciones de apoyos, recursos y profesionales capacitados en el área de la educación especial, en cambio la inclusión se fundamenta en un modelo donde toda la comunidad educativa y escolar están preparados para acoger la diversidad a través de un proceso socio comunitario. "La integración propone la adaptación curricular como medida de superación de las diferencias del alumnado con necesidades específicas de apoyo educativo; mientas que la inclusión propone un currículo común para todos en el que implícitamente vayan incorporadas esas adaptaciones" (PORRA, 2005, p. 50).

Revista @rquivo Brasileiro de Educação, Belo Horizonte, vol.2, num.3, jan-jun, 2014. 


\section{EDUCACIÓN INCLUSIVA, EDUCACIÓN DE CALIDAD}

El concepto de inclusión se origina en la Conferencia de 1990 de la UNESCO en Tailandia, donde se fomentó la idea de una Educación para Todos, como principio y política educativa, fundándose las primicias que van a regir la política práctica en la construcción de una educación inclusiva (KRICHESKY, 2006; PORRAS, 2005). Con ese acontecimiento, se inició un proceso de reflexión en respecto a la educación inclusiva, donde el principal objetivo es la unificación de personas con NEE en las aulas regulares y en la aceptación de las mismas. No obstante, es necesario reformular este proceso y posicionarse con un nuevo enfoque teórico que cimente razonablemente esta nueva perspectiva social, inclusiva y colaborativa de la educación.

Parafraseado a la Comisión de Expertos del MINEDUC, (2005) "La inclusión significa hacer efectivos para todos; el derecho a la educación, la igualdad de oportunidades y la participación" (CHILE, 2005). La educación inclusiva pretende una transformación de la educación general y de las unidades escolares, para que sean capaces de acoger y dar respuestas educativas a la diversidad de niños y niñas. La Inclusión fomenta el hecho de superar cualquier tipo de discriminación y exclusión que se pueda dar en el interior de las aulas escolares, partiendo de que muchos alumnos y alumnas no presentan igualdad de condiciones educativas, y muchas veces no se considera sus características personales.

Para que puedan surgir y desarrollarse escuelas inclusivas, debe haber un cambio profundo en los contextos educativos, partiendo de una realidad centrada en la homogeneidad a un entorno centrado en la diversidad. "Cuánto más inclusivas sean las escuelas comunes desde su origen, menos alumnos quedarán fuera de ellas y, por tanto, no será necesario integrarlos" (Informe de la comisión de expertos, (CHILE, 2004a, p.18).

Entonces, respondiendo a la visión inclusiva de las nuevas Políticas Educativas de una era globalizada, es pertinente crear elementos que unifiquen y potencien el trabajo de todos los actores que componen las distintas unidades educativas, generando Políticas Educativas de calidad y que respondan también a la equidad.

Para cumplir con este principio es necesario considerar una educación inclusiva, sustentada en que cada escuela presenta alumnos

Revista @rquivo Brasileiro de Educação, Belo Horizonte, vol.2, num.3, jan-jun, 2014. 
y alumnas que enfrentan los procesos académicos en diferentes condiciones, que dependen de su realidad personal y familiar, de sus capacidades innatas, de sus intereses y estilos de aprendizaje, propio de la diversidad (BOOTH, 2010).

Por lo tanto, los aprendizajes y desarrollo integral de los estudiantes de las unidades escolares, no solo dependen de las necesidades educativas propias e individuales, sino también dependen de las realidades que surgen en la vida diaria de cada escuela, relacionadas con la organización escolar, las estrategias de aprendizaje, los esfuerzos y metas de los docentes, las interacciones entre la familia, los intereses de los niños y niñas en sus propios procesos educativos. La forma y condición de abordar y vivir los procesos de enseñanza y aprendizaje como comunidad.

Por esta razón, el desarrollo de una educación inclusiva implica modificaciones en el propio sistema educativo, que den paso al surgimiento de políticas públicas en torno a este tema. Otorgando a los centro escolares los lineamientos teórico - prácticos a través de estrategias y herramientas que preparen a las comunidades educativas, a desarrollar un pensamiento intercultural, para realizar prácticas pedagógicas estratégicas en función de responder a los nuevos retos y desafíos de la educación inclusiva (KRICHESKY, 2006; CÉSPEDES, 2013; RAMON, 2010).

\section{DISEÑO METODOLÓGICO}

La investigación se sustenta dentro del paradigma cualitativo y se enmarca dentro de la Investigación Acción Participativa (LATORRE, 1997; COLMENARES; PIÑERO, 2008) la cual busca desarrollar el pensamiento reflexivo, crítico y emancipador para transformar y resolver problemas de una realidad social a través del trabajo constante, colaborativo e interactivo de todos los implicados. Permite relacionar desde una perspectiva empírica y conductual, la realidad curricular y práctica de los profesionales de la educación y las percepciones de las alumnas, sus padres y apoderados, frente a la labor pedagógica (ALVARADO; GARCÍA, 2008; GÓMEZ; ROQUET 2009).

\section{RESULTADOS PRELIMINARES}

Revista @rquivo Brasileiro de Educação, Belo Horizonte, vol.2, num.3, jan-jun, 2014. 
Durante el proceso de análisis se crearon categorías deductivas asociados al tema de la inclusión y las NEE. Como la presente investigación utiliza el paradigma de la Investigación Acción Participativa, se realizaron cuatro etapas o fases principales en el trabajo de campo, propias de este tipo de metodología. Las etapas corresponden a: Diagnóstico, Diseño de Planificaciones y Actividades Inclusivas, Aplicación de Actividades y finalmente la Evaluación y Difusión de los resultados.

En el presente artículo se presentará la etapa de Diagnóstico, ya que las otras tres fases están en proceso de análisis. En el periodo antes mencionado, se aplicaron distintas entrevistas y talleres a los informantes claves de este estudio, como profesores, especialistas, alumnas, padres y apoderados, con el objetivo de conocer sus percepciones y propuestas acerca de un modelo de atención inclusiva para la escuela.

El análisis estableció, en el caso de los docentes y profesionales especialistas, tres dimensiones: Sistema Educativo, Inclusión y Propuestas. En el caso de las alumnas y apoderados se determinaron cuatro categorías: Escuela y NEE, Exclusión, Inclusión y Propuestas.

Se desarrolló la creación de dos unidades hermenéutica a través del software Atlas ti, para cada grupo de informantes claves, la cual reúne las entrevistas y talleres que fueron analizados, para obtener finalmente los resultados de la etapa de diagnóstico para la investigación.

Dentro del primer análisis, correspondiente a la información obtenida de los profesores y especialistas, la dimensión Sistema Educativo, las categorías son las siguientes:

a) faltan Ajustes Curriculares: Los docentes señalan que no existen orientaciones o ajustes para responder pedagógicamente hacia las alumnas con NEE, lo que no permite la realización de prácticas inclusivas;

b) exigencias: este componente está directamente relacionada con los resultados académicos que se espera de las alumnas. Existe una serie de exigencias hacia los docentes y las alumnas. La evaluación estandarizada como el SIMCE, produce una presión e influencia en los docentes al momento de preparar y entregar sus contenidos a las estudiantes;

c) escuela-Familia-Estado: los docentes y profesionales manifestaron que no existe una conexión entre estos tres actores fundamentales

Revista @rquivo Brasileiro de Educação, Belo Horizonte, vol.2, num.3, jan-jun, 2014. 
para el desarrollo de una educación de calidad. Donde cada involucrado actúa por separado, la escuela como responsable directo de la formación educativa de la estudiante, antepone la integración por sobre la inclusión. En relación a la familia los profesores y especialistas indicaron que existe falta de apoyo y motivación por parte del hogar.

En la dimensión Inclusión, surgen cuatro categorías relacionados con las percepciones de los docentes y especialistas hacia las NEE y el desarrollo de prácticas pedagógicas inclusivas .Dentro de esta dimensión las subcategorías más relevantes que emergieron son:

a) letra Muerta: aquí aparece claramente la desorientación de los docentes y especialistas para abordar a los alumnos con NEE. Estos manifiestan que existe una realidad en el papel, pero en la práctica no se lleva a cabo;

b) competencias Docentes: en esta subcategoría, surge la formación y competencias docentes frente a las NEE y la inclusión educativa. Los docentes manifestaron la existencia de una ambigüedad del sistema educativo y falta de competencias, para abordar a los alumnos con NEE;

c) actividades Artísticas y Deportivas: esta subcategoría deja claramente establecido que los docentes consideran que las actividades artísticas y deportivas, favorecen las prácticas inclusivas, permitiendo a las alumnas expresarse con mayor libertad y cercanía hacia sus profesores, generando situaciones afectivas y de compañerismo entre docentes y alumnos.

En la dimensión Propuestas Inclusivas, surgen una serie de subcategorías donde los docentes y especialistas, opinan y proponen sus opiniones para el diseño de las actividades y transformación escolar. De esta dimensión, las ideas que emergieron de los discursos son:

a) equipo Directivo: en esta subcategoría los docentes y especialistas manifestaron el rol fundamental del equipo directivo al momento de concretar prácticas pedagógicas inclusivas, donde se propicie y potencie en la unidad educativa el trabajo colaborativo entre profesionales; 
Necesidades educativas especiales, elementos para una propuesta de inclusión educativa, a través de la investigación acción participativa. El caso de la escuela México

b) comunicación Especialistas y Profesores: mejorar la comunicación entre los especialistas y profesores de la comunidad educativa. A través de esta subcategoría se puede establecer la discordancia en el trabajo pedagógico y el apoyo de los especialistas de la escuela, ya que cada uno de los profesionales realiza el apoyo hacia las alumnas de manera aislada e individualista;

c) dinámicas de las Clases: en esta subcategoría emergen distintas propuestas específicas para cambiar y transformar directamente el trabajo en el aula y de esta forma concretar prácticas inclusivas. Los docentes en este caso, propusieron considerar en la realización de las actividades los estilos de aprendizaje, potenciar las habilidades como artísticas y deportivas, realizar mayoritariamente actividades grupales con las estudiantes y trabajar hacia una interconexión de las distintas disciplinas de estudio;

d) finalmente, en análisis, correspondiente a la información obtenida de las alumnas, padres y apoderados, las dimensiones que se desglosaron fueron:

e) escuela y NEE: En este caso, se desprende la percepción de los apoderados y las alumnas frente a la atención y acogida que brinda la Escuela a las alumnas con NEE, predominando códigos como falta de comunicación y actitudes de los profesores que no favorecen la inclusión;

f) exclusión: Existen en las aulas situaciones de exclusión hacia las alumnas con NEE, asociadas a las prácticas homogéneas que predominan en las actividades diarias;

En la subcategoría exclusión, nos encontramos con aspectos relevantes como que no existen progresos académicos relevantes en las educandas con NEE, esto hecho trae consigo una serie de elementos como la categorización y estigmatización para dichas alumnas, la falta de incentivos académicos. Además de problemas de disciplina y responsabilidad asociados a las NEE;

g) en relación a la subcategoría de inclusión, los apoderados y alumnas manifestaron que el hecho de contar con alumnas $y$ compañeras que presenten NEE, permite desarrollar el sentido de respeto por la diversidad, aportando consigo beneficios positivos para todo el grupo de estudiantes;

h) Finalmente, en la subcategoría propuestas, los elementos más relevantes que surgieron son: mejorar las dinámicas de las clases,

Revista @rquivo Brasileiro de Educação, Belo Horizonte, vol.2, num.3, jan-jun, 2014. 
apoyo por parte de las especialistas en el aula común, trabajo personalizado, trabajo con alumnas tutoras, técnicas de estudio, entre otros.

\section{CONCLUSIONES}

Se logra comprender en esta etapa preliminar de la investigación, elementos fundamentales para la base de la construcción de la propuesta de inclusión educativa para las alumnas con NEE de la Comunidad Escolar de la Escuela México de la Ciudad de Valdivia. Al respecto y a partir del establecimiento de categorías y códigos para llevar a cabo el análisis se identificaron las percepciones hacia la inclusión y las NEE por parte de los docentes, especialistas, alumnas, padres y apoderados.

En el caso de los profesores y especialistas, se pudo determinar de acuerdo a las percepciones de los entrevistados que el Sistema Educativo actual, presenta una serie de elementos que dificultan las prácticas inclusivas en las aulas escolares. A raíz de esto es que se necesita fomentar una cultura inclusiva de enseñanza, que comience con hechos o situaciones locales y particulares, para posteriormente abordar contextos globales, así de esta manera, educar para y hacia la diversidad, impulsando el trabajo colaborativo y participativo de todos los implicados.

Los puntos más relevantes que se pueden destacar, son la falta de orientaciones didácticas y curriculares para acoger a la diversidad, situación que está predeterminada por el contexto escolar y educativo que viven a diario los docentes y especialistas. Esta realidad está predeterminada por el propio Sistema Educativo existente, que dificulta la realización de prácticas inclusivas.

Por otra parte, los docentes no están familiarizados con el tema de la inclusión, manifestando que existen políticas que no se llevan a cabo en la realidad y solo están escritas en el papel, primando las prácticas pedagógicas conductistas y homogéneas, por ser más fácil de realizar y aplicar en sus actividades diarias.

Conjuntamente, esta realidad se asocia igualmente a la falta de competencias docentes en el tema de las NEE y la inclusión educativa. Los profesores señalan que no fueron preparados en su formación inicial, en la atención de estudiantes que presenten NEE, y durante el ejercicio

Revista @rquivo Brasileiro de Educação, Belo Horizonte, vol.2, num.3, jan-jun, 2014. 
de su profesión, no han recibido capacitaciones u orientaciones para implementar prácticas más acorde a la diversidad de estudiantes.

Así mismo, las principales propuestas que se pueden rescatar en esta etapa de la investigación, según las percepciones de los docentes y especialistas, es fomentar desde el equipo directivo, una cultura inclusiva de enseñanza y el trabajo colaborativo de los involucrados. Logrando de esta forma, mejorar la comunicación entre profesores y especialistas, para optimizar el trabajo en el aula y así, perfeccionar las dinámicas de las clases, fomentando la interdisciplinariedad de enseñanza.

Por otra parte, las propuestas que manifestaron los apoderados para lograr prácticas pedagógicas más inclusivas, destacan el cambiar las dinámicas de las clases y sugieren distintos elementos como, que exista una relación entre las distintas asignaturas, a través de la interdisciplinariedad de enseñanza. Realizar actividades recreativas, donde las niñas puedan potenciar sus habilidades. Fomentar el trabajo con alumnas tutoras y actividades grupales, para de esta manera promover el trabajo colaborativo y solidario entre compañeras. Además de fomentar el respeto por la diversidad.

En cuanto a las percepciones y propuestas de las alumnas, emergen como puntos relevantes el hecho de la categorización y estigmatización hacia las alumnas con NEE, existiendo una diferenciación negativa hacia dichas estudiantes, concretándose en hechos como actitudes poco constructivas, por parte de algunos docentes y rechazo de algunas compañeras de curso. Además, las alumnas manifestaron una falta de comunicación, incomprensión y carencia de afectos, por parte de sus hogares, hecho que notoriamente determina el logro de aprendizajes significativos en las estudiantes.

A su vez, la escuela está determinada por situaciones como la actitud de los docentes, presentando condiciones negativas y positivas. Las negativas relacionadas coincidentemente con lo manifestado por los profesores, especialistas y apoderados, con la categorización y estigmatización que existe hacia las alumnas con NEE, por no lograr avances académicos sobresalientes, lo que afecta considerablemente la autoestima de dichas alumnas.

Al mismo tiempo, las alumnas manifestaron situaciones positivas por parte de sus profesores, indicando que los docentes se demuestran dispuestos y propositivos para explicar algún determinado contenido.

Estas dos realidades, las actitudes positivas y negativas de 
los docentes, generan un clima en el aula que puede ser beneficio o pernicioso para los implicados, tanto estudiantes como profesionales. Por lo tanto, es importante reflexionar sobre esta situación, tanto a nivel de aula, como a nivel de comunidad educativa, para de esta manera generar mejoras en relación a un clima afectivo en el aula que sea beneficioso, tanto para las estudiantes, como para los profesionales que se desempeñan a día a día en las aulas.

En el caso de las propuestas que manifestaron las alumnas, para mejorar hacia una cultura inclusiva, al igual que en los casos anteriores, se relacionan con cambiar las dinámicas de las clases, por medio de fomentar en las estudiantes el gusto por el aprendizaje, a través de la utilización de distintas estrategias metodológicas como el trabajo con las manos o táctil, donde hechas puedan tocar y crear situaciones de aprendizaje.

Al igual que en las propuestas sugeridas por los docentes, especialistas y apoderados, se repite el hecho de realizar actividades con alumnas tutoras y trabajos grupales, fomentado la colaboración y sentido solidario. Paralelamente, proponen realizar un trabajo personalizado, donde el docente explique puesto por puesto, para una mejor comprensión de los contenidos.

Otra propuesta es potenciar en el establecimiento el uso de tecnologías, herramienta que motiva y contextualiza a las alumnas, por estar día a día conviviendo con recursos tecnológicos.

Finamente, otro elemento relevante y que se repite como propuesta tanto por los docentes, especialistas, como por los apoderados y alumnas, es el hecho de concretar una forma de evaluación más eficiente, para de esta forma mejorar los resultados académicos de las alumnas.

Se establece entonces la necesidad de fomentar en el establecimiento en estudio, una reflexión permanente sobre la cultura escolar, sus modelos organizativos y de igual forma, las prácticas pedagógicas utilizadas en la realidad cotidiana en el interior del centro educacional. De esta manera, se podrá indagar e identificar las actitudes y expectativas que presenten los docentes y especialistas, sobre las alumnas con NEE. Los cuales ya no pueden continuar sustentados en una baja expectativa pedagógica, respecto a sus logros académicos, sino que requieren respuestas innovadoras construidas de manera colaborativa, en espacios de desarrollo profesional inclusivo. 


\section{RESUMO}

O presente estudo visa o desenvolvimento, implementação e desenvolvimento de um modelo de educação inclusiva através de Pesquisa Acção Participativa para a comunidade escolar do Escola México de Valdivia Chile. A proposta visa criar, através de diversos facilitadores de salas de aula inclusivas como estilos de aprendizagem, a colaboração de colaboração entre pares de trabalho entre os profissionais, workshops para pais, entre outros componentes que possam surgir no decorrer da investigação.Assim, permitindo a inclusão de alunos com necessidades educacionais especiais na pesquisa estabelecimento. Além disso, através desta proposta educativa, tem como objetivo promover o desenvolvimento de uma cultura inclusiva de ensino em toda a comunidade da educação por meio de reflexão, avaliação e feedback constante dos participantes possuem a metodologia pesquisa-ação.

Palavras-finques: Inclusão. Integração. Diversidade.Necessidades Educativas Especiais.

\section{Special Educational Needs, elements for inclusion education} proposal through participatory action research. The case of México school

\section{ABSTRACT}

The current study aims to the elaboration, implementation and development of an inclusive model of education through Participatory Action Research for the Mexico school community in Valdivia Chile. The proposal is to create, through several facilitators of inclusive classrooms, such as learning styles, collaborative work peer, collaboration between professionals, parent workshops, among other components, that arise in the course of the investigation. Thus allowing the inclusion of pupils with special needs education, on the study place. In addition, through this educational proposal, it is pretend to promote the development of an inclusive culture of teaching throughout the education community based on reflection, evaluation and constant feedback from the participants, distinctive feature of methodology of Action Research. 
Keywords: Education Inclusion. Integration. Diversity. Special Needs Education.

\section{REFERÊNCIAS}

ALVARADO, Lusmidia; GARCIA, Margarita. Características más relevantes del paradigma socio-crítico: su aplicación en investigaciones de educación ambiental y de enseñanza de las ciencias realizadas en el doctorado de educación del Instituto Pedagógico de Caracas. Revista Universitaria de Investigación, Caracas, Venezuela, año 9, no 2 , 2008.

BOOTH, Tony. Inclusion in education: participation of disabled learners, en executive summaries, Education for All 2000 Assessment. In: FORUM INTERNATIONAL CONSULTATIVE ON EDUCATION. Executive summaries, Education for All. Paris: UNESCO, 2000. p 43-50.

Disponible en: <http://unesdoc.unesco.org/images/0012/001234/123486e.pdf> Acceso el: 02 junio 2010.

CESPEDES, Amanda et al. Camino a la escuela inclusiva: libro guía para educadores: trastornos del desarrollo desde las neurociencias aplicadas a la educación. Chile: Fundación Mirame, 2013.

\section{CHILE. Ley de Integración Social de las Personas con}

Discapacidad. 1994. Disponible en: <http://www.mineduc.cl/biblio/ documento/200810271405400. Ley_de_Integracion_Social_de_las_ Personas_con_Discapacidad-19.284.pdf > Acceso el: 02 junio 2010.

CHILE. Ley No 20422. Establece Normas sobre Igualdad de Oportunidades e Inclusión Social de Personas con Discapacidad. 2010. Disponible en: < http://www.senadis.gob.cl/pag/177/620/ley_n20422> Acceso el: 02 junio 2010.

CHILE. El Ministerio de Educación. Comisión de expertos en educación especial: nueva perspectiva y visión de la educación especial. Santiago de Chile: MINEDUC, 2004a.

CHILE. El Ministerio de Educación. Estudio a nivel muestral sobre la calidad del proceso de integración educativa. Santiago de Chile: MINEDUC, 2004b.

CHILE. El Ministerio de Educación. Pólitica Nacional de Educación Especial: nuestro compromiso con la diversidad. Santiago de Chile: MINEDUC, 2005. 
Necesidades educativas especiales, elementos para una propuesta de inclusión educativa, a través de la investigación acción participativa. El caso de la escuela México

COLMENARES, E. A. M.; PIÑERO M. La Investigación Acción. Una herramienta metodológica heurística para la comprensión y transformación de realidades y prácticas socio-educativas. Laurus, Venezuela, v. 14, n² 27, p. 96-114, mayo/ago. 2008.

FUNDO DAS NAÇÕES UNIDAS PARA A INFÂNCIA. Ciclo de debates: desafíos de la política educacional "claves de la inequidad en la educación básica". Chile y Uruguay: Oficina de Área para Argentina, 1999.

GODOY, M. P.; MEZA, M. L.; SALAZAR, A. Antecedentes históricos, presente y futuro de la educación especial en Chile. Ministerio de Educación, Programa de Educación Especial, 2004. Disponible en: <http://www.mineduc.cl/biblio/documento/Antec_ historicospresenteyfuturo_EducEsp2004.pdf> Acesso em: GÓMEZ, David Rodríguez; ROQUET, Jordi Valldeoriola. Metodología de la Investigación. Universitat Oberta de Catalunya, 2009.

KRICHESKY, M. Escuela y comunidad: desafíos para la inclusión educativa. Buenos Aires. Argentina: Ministerio de Educación, Ciencias y Tecnología de la Nación, 2006.

KEMMIS, S.; MCTAGGART, R. Cómo planificar la investigación-acción. Barcelona: Laertes, 1988.

LATORRE, A. La investigación: acción, conocer y cambiar la práctica educativa, Barcelona España: Ed. Graó, 2003.

LATORRE, A. Bases metodológicas de la investigación educativa. Barcelona, España: Ed. Nortado, 1997.

ORGANIZAÇÃO PARA A COOPERAÇÃO E DESENVOLVIMENTO ECONÓMICO. Panorama de la Educación. Indicadores de la OCDE 2009. Resumen en Español. Disponible en: <http://www.oecd.org/ dataoecd/1/8/43654730.pdf> Acesso em: 14 dez. 2014.

PORRAS, A. El valor de la educación intercultural. Madrid, España: Edit. Visión Libros, 2005.

RAMON, María Rosa Rosselló. El reto de planificar para la diversidad en una escuela inclusiva. Revista Iberoamericana de Educación, n 51/4, Feb. 2010.

TACSAN, Mayra Achio. Etica de la Investigación en ciencias sociales: repesando temas viejos. Revista Perspectivas Bioéticas, Buenos Aires Argentina, año $8 \mathrm{n}^{\circ} 15$ Bis, $2^{\circ}$ sem., 2003.

Revista @rquivo Brasileiro de Educação, Belo Horizonte, n.2, vol.1, jan - jun, 2014 
UNITED NATIONS EDUCATIONAL SCIENTIFIC AND CULTURAL

ORGANIZATION: Educación para todos: cumplir nuestro compromisos comunes. In: FORO MUNDIAL SOBRE LA EDUCACIÓN. Marco de Acción. Dakar, Senegal: 2000.

UNITED NATIONS EDUCATIONAL SCIENTIFIC AND CULTURAL

ORGANIZATION. Cada escuela es un mundo: un mundo de diversidad. Experiencias de integración educativa. Santiago de Chile: 2003.

UNITED NATIONS EDUCATIONAL SCIENTIFIC AND CULTURAL ORGANIZATION. Temario abierto sobre educación inclusiva. materiales de apoyo para responsables de políticas educativas. Santiago de Chile: OREALC-UNESCO, 2004.

UNITED NATIONS EDUCATIONAL SCIENTIFIC AND CULTURAL ORGANIZATION. Declaración de Salamanca y Marco de Acción para las necesidades educativas especiales: acceso y calidad. Salamanca España: 1994. 